\title{
Corrigendum
}

\section{Corrigendum to "Evans Blue Dye: A Revisit of Its Applications in Biomedicine"}

\author{
Linpeng Yao $\mathbb{D}^{1},{ }^{1}$ Xing Xue $\mathbb{D}^{\circ},{ }^{1}$ Peipei Yu, ${ }^{1,2}$ Yicheng Ni $\mathbb{D},^{3}$ and Feng Chen $\mathbb{D}^{1}$ \\ ${ }^{1}$ Department of Radiology, The First Affiliated Hospital, Zhejiang University School of Medicine, 79 Qingchun Road, Hangzhou, \\ Zhejiang 310003, China \\ ${ }^{2}$ Department of Radiology, Sanmen County People's Hospital, Sanmen, Zhejiang 317100, China \\ ${ }^{3}$ Radiology Section, University Hospitals, University of Leuven, 3000 Leuven, Belgium \\ Correspondence should be addressed to Feng Chen; chenfenghz@zju.edu.cn
}

Received 21 August 2021; Accepted 21 August 2021; Published 6 September 2021

Copyright $\odot 2021$ Linpeng Yao et al. This is an open access article distributed under the Creative Commons Attribution License, which permits unrestricted use, distribution, and reproduction in any medium, provided the original work is properly cited.

In the article titled "Evans Blue Dye: A Revisit of Its Applications in Biomedicine" [1], the authors mistakenly cited Yet et al. instead of Jahr J S et al. on page 3. Thus, the sentence "Yet et al. [47] developed a novel approach using HBOC in rabbits in 2001, which is inexpensive and not time-consuming, while Baby et al." should be corrected as "Jahr J S et al. [47] developed a novel approach using HBOC in rabbits in 2001, which is inexpensive and not time-consuming, while Baby et al." and the corresponding reference should be corrected as follows [2]:

[47] Jahr J S, Lurie F, Xi S, et al. “A novel approach to measuring circulating blood volume: the use of a hemoglobin-based oxygen carrier in a rabbit model", Anesthesia \& Analgesia, 2001, 92(3):609-614.

\section{References}

[1] L. Yao, X. Xue, P. Yu, Y. Ni, and F. Chen, "Evans blue dye: a revisit of its applications in biomedicine," Contrast Media and Molecular Imaging, vol. 2018, Article ID 7628037, 10 pages, 2018.

[2] J. S. Jahr, F. Lurie, S. Xi et al., "A novel approach to measuring circulating blood volume: the use of a hemoglobin-based oxygen carrier in a rabbit model," Anesthesia \& Analgesia, vol. 92, no. 3, pp. 609-614, 2001. 